\title{
A 68-year-old with cranial nerve neuropathies and a troponin rise
}

\author{
Authors: Luke Bennett ${ }^{A}$ and Javed Iqbal ${ }^{B}$
}

In this case study, we summarise the inpatient investigations and management of a 68-year-old woman with Takotsubo cardiomyopathy secondary to a Varicella zoster encephalitis and the difficulties inherent with making this diagnosis. She presented with evolving cranial nerve neuropathies, which started with a vagal nerve mononeuritis and eventually included left-sided sensorineural hearing loss and a facial nerve palsy. These symptoms were concomitant with a variety of cardiac abnormalities, including fast atrial fibrillation and electrocardiographic changes. We summarise some of the current understanding of Takotsubo cardiomyopathy and the criteria for its diagnosis. Although left ventricular apical ballooning has been described in association with severe infections and states of high stress, we have not seen it reported in association with a Varicella zoster encephalitis.

KEYWORDS: Cranial mononeuropathy, cranial nerve neuropathies, encephalitis, Takotsubo cardiomyopathy, troponin rise, Varicella zoster

\section{Introduction}

Takotsubo cardiomyopathy (also known as apical ballooning syndrome or stress-induced cardiomyopathy) is an acute clinical syndrome characterised by transient regional systolic dysfunction of the left ventricle. This syndrome can clinically mimic acute coronary syndrome in terms of clinical presentation and electrocardiographic changes; crucially, however, there is no angiographic evidence of an obstructing coronary disease. ${ }^{1}$ Takotsubo cardiomyopathy can be triggered by systemic responses to physical and emotional stress and sepsis is a strong predictor of left ventricular apical ballooning. ${ }^{2}$ We report a case of this cardiac syndrome developing in response to viral encephalitis.

\section{Case presentation and initial management}

A 68-year-old woman with no significant past medical history presented to the ear, nose and throat team with a 4-day history

Authors: ${ }^{\text {A }}$ core medical trainee, Dorset County Hospital, Dorchester, UK; ${ }^{B}$ consultant interventional cardiologist, Dorset County Hospital, Dorchester, UK of dysphagia with hoarseness and subsequent loss of voice.

Nasolaryngoscopy demonstrated paralysis of her left soft palate and vocal cord, suggesting a vagus nerve cranial mononeuritis. She also reported palpitations occurring over the previous 24 hours. An electrocardiogram (ECG) revealed new fast atrial fibrillation at $130 \mathrm{bpm}$. Bloods revealed raised inflammatory markers with a white blood count (WBC) of $11.2 \times 10^{9} / \mathrm{L}$ and a C-reactive protein of $151 \mathrm{mg} / \mathrm{L}$. A high sensitivity troponin was elevated at 2,577 ng/L, rising to 3,808 ng/L after 3 hours. She was referred to the cardiology team.

Her chest radiograph showed no obvious pneumonic process or focal lesion that could account for the recurrent laryngeal nerve. Repeat ECG with rate control showed mild anterolateral ST-elevation (Fig 1) followed by widespread anterolateral T-wave inversion persisting throughout admission. She was treated empirically as an acute coronary syndrome with dual antiplatelet therapy. In view of the possible left sided 10th nerve palsy a magnetic resonance imaging (MRI) head was performed, showing no focal cerebral, cerebellar or brainstem lesion. An echocardiogram performed 24 hours after admission showed apical akinesis with mild to moderate left ventricular systolic impairment.

The differential diagnosis was between a primary ischaemic cardiac problem leading to dysrhythmia and subsequent small brainstem embolus or a systemic inflammatory process causing a cranial nerve neuropathy and cardiac pathology (viral myocarditis or vasculitis)

\section{Case progression}

An episode of 'coffee-ground vomiting' delayed coronary angiography; fortunately, no evidence of recent upper gastrointestinal bleeding was seen on oesophagogastroduodenoscopy.

Her cranial nerve neuropathies progressed with left-sided sensorineural hearing loss and lower motor neurone facial palsy. Repeat computerised tomography head showed no evidence of evolving intracranial haemorrhage. Neurology review suggested repeat MRI with fluid attenuation inversion recovery and diffusion-weighted imaging with magnetic resonance cerebral and carotid angiography - demonstrating little difference compared with the old MRI. Serum Lyme IgG and IgM antibody, rheumatoid factor, anti-nuclear antibodies, perinuclear and cytoplasmic antineutrophil cytoplasmic antibodies and serum angiotensin-converting 
Fig 1. The patient's initial electrocardiogram, showing anterolateral ST changes.

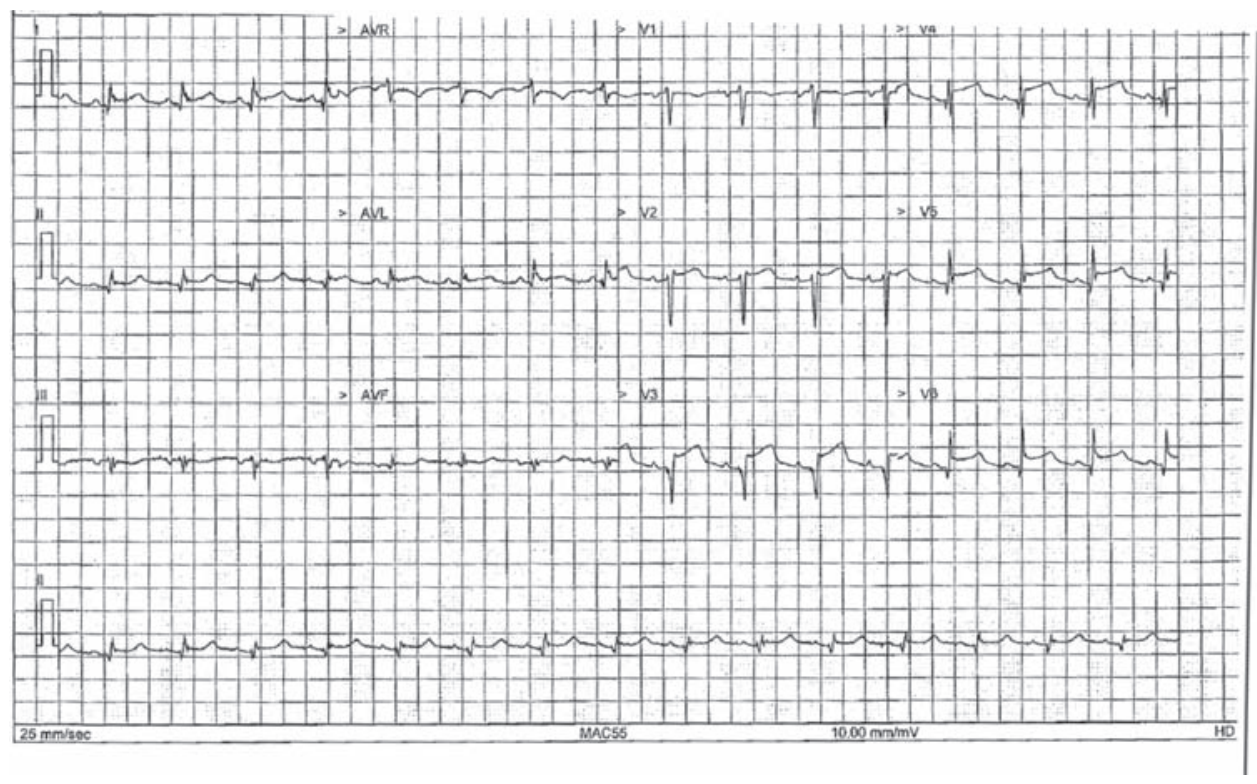

enzyme were negative, as were tests for hepatitis B and C, HIV and syphilis.

A lumbar puncture revealed a lymphocytic pleocytosis (WBC $\left.65 \times 10^{6}\right)$. No organisms were seen. Cerebrospinal fluid (CSF) protein was normal $(0.43 \mathrm{~g} / \mathrm{L})$. CSF glucose was $4.3 \mathrm{mmol} / \mathrm{L}$ with a paired serum glucose of $7.3 \mathrm{mmol} / \mathrm{L}$. CSF cytology was negative for malignant cells. The CSF was sent for viral polymerase chain reaction (PCR) and a positive result for Varicella zoster virus was discovered.

She was diagnosed with Varicella zoster encephalitis causing cranial nerve polyneuropathies and commenced on intravenous aciclovir. Repeat lumbar punctures after 4 and 10 days of acyclovir treatment demonstrated an improving lymphocytosis and repeat PCR was no longer positive for Varicella zoster.

\section{Outcome}

Following treatment, the patient's swallow, voice and hearing improved; however, persistent left-sided lower motor neurone facial weakness remained. It is unclear whether this will improve or whether there will be a permanent deficit. From a cardiac perspective, she had persistent T-wave inversion but no chest pains or further runs of atrial fibrillation.

The planned coronary angiogram showed normal coronary arteries and her echocardiograms were reviewed again by a consultant cardiologist who saw evidence of apical ballooning and diagnosed a Takotsubo cardiomyopathy in response to her Varicella zoster virus encephalitis.

She was discharged with ramipril and ivabradine and completed a further 7 days of valaciclovir in addition to the 14 days of intravenous aciclovir she received.

Apical ballooning in Takotsubo is a transient process and the left ventricular hypokinesia should resolve with the improvement in her systemic illness. She is being monitored with serial echocardiograms in the community. The speed of resolution of the ballooning has been debated in the literature.

\section{Discussion}

Stress cardiomyopathy is an increasingly recognised phenomenon since its first description in Japan in 1990. It appears to be more prevalent in women, especially if postmenopausal, and predominantly affects the left ventricular mid-cavity and apex. Transient apical ballooning has been shown to be a relatively common echocardiographic finding in critically unwell patients with no prior cardiac history in intensive care. ${ }^{3}$

The Mayo Clinic have proposed four stipulations for Takotsubo diagnosis $;{ }^{4}$ however, no official consensus has been reached:

1 transient dyskinesia of the left ventricular mid segment + /apical involvement - the wall motion abnormality extending beyond the territory of a single coronary artery

2 no evidence on coronary angiography of obstructive lesion or acute plaque rupture

3 new changes on electrocardiography (eg ST-elevation and/ or T-wave inversion)

4 absence of other causative pathologies.

Table 1. Reference ranges for laboratory investigations

\section{Laboratory investigation}

Serum white blood count

C-reactive protein

High sensitivity troponin

Serum glucose

Cerebrospinal fluid white

Cerebrospinal fluid glucose

Cerebrospinal fluid protein blood count

\section{Reference range}

$4.5-11.0 \times 10^{9} / \mathrm{L}$

$<5 \mathrm{mg} / \mathrm{L}$

$<15 \mathrm{ng} / \mathrm{L}$

$3.9-5.5 \mathrm{mmol} / \mathrm{L}$

0-5 mononuclear white blood cells

$2.5-4.4 \mathrm{mmol} / \mathrm{L}$

$0.15-0.6 \mathrm{mg} / \mathrm{mL}$ 
This patient fulfilled all criteria, possessing typical echocardiographic appearances and normal coronary arteries on angiography. Her troponin was elevated, which is a common finding in Takotsubo ( $86.2 \%$ of patients). ${ }^{5}$ Clinically, it is difficult to differentiate this presentation from that of an acute coronary syndrome - certainly the diagnosis of this patient was delayed because of her episode of 'coffee-ground vomiting', which delayed angiography. A cardiac MRI may have helped differentiate between an acute ischaemic event, myocarditis and true apical ballooning; however, it was not available acutely.

A literature review suggests Takotsubo has previously been described in association with limbic encephalitis or in patients where the encephalitis has caused severe cerebral oedema and hydrocephalus. We have not seen it described in association with Varicella zoster encephalitis. The pathogenesis of this phenomenon is still uncertain. High levels of circulation catecholamines can trigger apical cardiodepression and are postulated to be an underlying causative mechanism. ${ }^{6}$ This may fit with the established association with physical (eg sepsis, critically unwell) and emotional stressors.

There is no definitive guidance for therapies (pharmacological and non-pharmacological) for management of Takotsubo and recommendations are based on treating the complications of the disease, such as systolic dysfunction and arrhythmias.

In conclusion, Takotsubo cardiomyopathy should be included in the differential diagnosis of patients with ECG changes, left ventricular regional wall motion abnormality and absence of obstructive coronary artery disease.

\section{Conflicts of interest}

The authors have no conflicts of interest to declare.

\section{Acknowledgements}

Consent was obtained from the patient for the publication of the clinical details and images in this article.

\section{References}

1 Tsuchihashi K, Ueshima K, Uchida T et al. Transient left ventricular apical ballooning without coronary artery stenosis: a novel heart syndrome mimicking acute myocardial infarction. Angina PectorisMyocardial Infarction Investigations in Japan. J Am Coll Cardiol 2001;38:11-8.

2 Park JH, Kang SJ, Song JK et al. Left ventricular apical ballooning due to severe physical stress in patients admitted to the medical ICU. Chest 2005;128:295-302.

3 Bybee KA, Kara T, Prasad A et al. Systematic review: transient left ventricular apical ballooning: a syndrome that mimics ST-segment elevation myocardial infarction. Ann Intern Med 2004;141:858-65.

4 Madhavan M, Prasad A. Proposed Mayo Clinic criteria for the diagnosis of Tako-Tsubo cardiomyopathy and long-term prognosis. Herz 2010;35:240-3.

5 Prasad A, Lerman A, Rihal CS. Apical ballooning syndrome (TakoTsubo or stress cardiomyopathy): a mimic of acute myocardial infarction. Am Heart J 2008;155:408-17.

6 Pilgrim TM, Wyss TR. Takotsubo cardiomyopathy or transient left ventricular apical ballooning syndrome: a systematic review. Int $J$ Cardiol 2008;124:283-92.

Address for correspondence: Dr Luke Bennett, Dorset County Hospital, Williams Avenue, Dorchester, Dorset DT1 2JY, UK. Email: lukealexbennett@gmail.com 